\title{
The Uncertainty Principle in Modern Physics
}

GOMEWHERE in the literature of the theory of relativity occurs the statement: "The theory of relativity is physics, and we render it no good service by too hastily attributing to it a philosophical or indeed a speculative metaphysical meaning". Prof. C. G. Darwin, in his Friday evening discourse before the Royal Institution on Feb. 26, did not quite make the same statement about the uncertainty principle, but he stressed its physical side and had only a word or two to say on its other aspects. Prof. Darwin believes that the question of human free will is not touched by recent developments in physics. It is doubtful if any of the old deterministic descriptions of Nature were antagonistic to the doctrine of human free will, for it is a presumption to suppose that the organic world is controlled only by the laws of physics and chemistry. Since the Heisenberg uncertainty relations are also laws of the inorganic world, it is again a presumption to suppose that they control those activities in which human beings feel that they have freedom of choice. What the new principle does is point out to the 'die-hard' determinist that there is nothing in physics which makes the idea of free will absurd.

Prof. Darwin's lecture was a very clear exposition of the principle, and those who were not present to hear it will profit by reading the printed version. He gives an excellent illustration of the completely wave-like character of particles. If electrons are shot through two small holes situated close together in a screen, they produce an interference pattern on a photographic plate placed to receive them on the other side. If one hole is covered so that a pin-hole camera results, the electrons passing through the remaining hole produce general fogging of the plate. This is an experiment which can be performed in thought only ; it is given as a type and contains the essential point of other practical experiments. It brings out the fact that these showers of particles have more than a particle aspect; they are also waves.

The Heisenberg uncertainty principle is the expression of our inability to locate a particle, for example, an electron, and to measure its momentum accurately at the same time. Heisenberg appears to take the view that processes in Nature are discontinuous. Other exponents of the theory assume that the laws of Nature are average or probability laws. They go so far as to say that the principle of causality no longer holds in the old sense in which that principle was applied.

Another possible conclusion is that the new principle is a consequence of our predilection for the description of phenomena in space-time, and that a wider background than that provided by the four variables $x, y, z$, and $t$ would make the principle unnecessary. This question and the attempt to discover a unitary method of description in physics are closely associated.

The theory of relativity stresses the lack of union between gravitational and electromagnetic pheno- mena, and since its advent several attempts have been made to unite these two domains. Weyl, Eddington, and Einstein have all succeeded in doing so ; but quantum phenomena stand aside from their theories in the same way that electromagnetism stood aside from Einstein's theory of gravitation.

Dirac's equations, especially in Darwin's form, point to a remarkable analogy between the quantum and electromagnetic theories, and it has been shown that these fundamental equations of the new quantum theory are expressible in the form of a vanishing divergence, when the geometrical background is that afforded by the use of five variables. This at once suggests the possibility of union of all the three domains, and the theory has been successfully developed.

We are here concerned with one result arising from this unitary theory, namely, the fundamental importance of the length $h / m_{0} c$, associated with any particle of mass $m_{0}$.

The theory shows that Planck's assumption of the existence of a quantum of action and the WilsonSommerfeld relations both lead to the result that no length less than $h / m_{0} c$ is detectable in association with the particle.

The principle, stated in a slightly different form, has been called the principle of 'minimum proper time'. The length in this case is measured along the track of the particle in space-time. When interpreted in terms of ordinary space and time, it means that in the case of a particle moving with velocity $v$, the smallest detectable length is :

$$
\Delta l=\frac{h}{m_{0} c} \frac{\beta}{\sqrt{1-\beta^{2}}} \text { where } \beta=v / c ;
$$

and the smallest detectable interval of time is :

$$
\Delta t=\frac{h}{m_{0} c} \cdot \frac{1}{\sqrt{1-\beta^{2}}} .
$$

This means that there is always an uncertainty in the position of the particle of amount $\Delta l$. The amount increases with the velocity, and if the velocity of light could be attained, it would be impossible to locate it at all. Like a monochromatic wave, it might be said to occupy all space. This principle of uncertainty was also discovered by Ruark in 1928 in a different form and by a different method, but his statement of it is evidently the expression of the same principle. Since that time it has been frequently rediscovered and discussed.

In spite of the fundamental difference between this principle and that of Heisenberg, it can be shown that it leads to his relations when the distances concerned are small. A very interesting result follows from an application to atomic orbits. No atomic orbit can be shorter than $\Delta l$, otherwise we could detect its existence through the spectrum of the atom in which it occurs. It follows from this that there is a maximum atomic number, and calculation shows the number to be 97 . The prin- 
ciple admits the possible existence of heavier nuclei, but declares that it is impossible for atoms of higher number, built on the Rutherford model, to exist with their full complement of electrons. If the laws we discover in physics hold in all parts of the universe, we deduce that there is this definite limit to the number of elements existing. This is an interesting conclusion, and it is remarkable that the number falls so close to the number of elements at present known, namely, 92. The principle behaves here as a condition of stability, and it may be that elements of higher number than 92 are extremely unstable, and now remain only in minute quantities.
This question of the maximum number of elements possible has been discussed by V. V. Narliker in Nature for March 12, p. 402 ; 1932. The notation and method of Sir Arthur Eddington have been applied, and from considerations of invariance the maximum number obtained is 92 .

The unitary theory and uncertainty principle deduced from it indicate that observers in space and time will discover both space and time are discontinuous, for their measurements of intervals of length and time will always be in integral multiples of $\Delta l$ and $\Delta t$. The method of arriving at this conclusion indicates that this discontinuity underlies the old quantum theory.

H. T. Flint.

\section{Sir Charles Parsons and his Work}

" GAVERY, Newcomen, Smeaton, the famous Watt, Woolf, Trevithick, and some other English engineers were the veritable creators of the steam engine," said Sadi Carnot in his essay of 1824, while a year ago the Engineer, on the occasion of the death of Sir Charles Parsons, remarked that " to Great Britain belongs the credit for the origination of nearly all the fundamental inventions for the production and industrial utilisation of heat energy".

Although during the nineteenth century many improvements in heat engines, more especially in internal combustion engines, were made abroad, it remains true that it is in the records of the British Patent Office that most of the outstanding inventions made in connexion with steam engines and steam turbines are to be found. With the steam turbine will ever be associated the name of Sir Charles Parsons, who, as Sir Alfred Ewing said when lec. turing on the work of Parsons at the Royal Institution last November, "was incomparably the most illustrious and most revolutionary engineer of his time ". In view of the long and close friendship of Sir Alfred with Parsons, it was almost inevitable that on the occasion of this lecture, an account of which has recently been published, he should pay a tribute to him not only as an engineer but also as a friend. He therefore addressed his remarks not so much to those familiar with the steam turbine and its development, as to those who wished to know more of what Parsons did and how he did it, and what manner of man he was that he should be ranked so confidently among famous men.

Allowing for differences in time and circumstances, there is a remarkable similarity between the work done by Watt and by Parsons, and what the latter said of his great forerunner might well be applied to himself. When delivering the second Joule memorial lecture to the Manchester Literary and Philosophical Society in 1922, Parsons said: "James Watt's conclusions appear to have been the result of close and patient reasoning of a mind endowed with extraordinary powers of insight into physical questions, and of drawing sound practical conclusions from numerous experiments devised to throw light on the subject under consideration".

In his early surroundings, Parsons was even more fortunate than Watt, and his boyhood was spent in an atmosphere calculated to stimulate to the highest degree his interest in science and the mechanical arts. His father, by his construction of the famous reflecting telescope, had made Birr Castle the 'Mecca' of astronomers all over the world, while his mother possessed manipulative skill of no mean order. His tutors, too, were men of the highest calibre. In its influence on its various members, the home at Birr may well be compared to that of the Darwins at Downe. From it, Parsons emerged one of the most industrious of men, but also one of the shyest, and the effects of his unconventional boyhood remained stamped on him for life. It was his natural reserve and modestycharacteristics possessed by few inventors-which puzzled strangers. "His hesitating, fragmentary speech, his passive hand-shake, his somewhat shrinking manner," said Sir Alfred, "gave at first an impression of ineffectiveness which completely belied the latent force, the precision of purpose, the resolution that treated obstacles only as incentives to further effort, the fertility of invention that never accepted defeat."

Of Parsons' invention and development of the steam turbine, it may be said it was a piece of creative work comparable to that of a great writer or artist. Moreover, it was one for which the time was ripe, and which immediately and successfully fulfilled its purpose. The slow-speed reciprocating engines developed from the original inventions of Newcomen and Watt were ill adapted for driving the electric generators which were just coming into use when Parsons started work, and he was but one of many who attacked the problem of high speed. De Laval solved it one way and Parsons another. Thanks to the authorities at the Science Museum, Sir Alfred was able to exhibit at his lecture the historic turbo-generator Parsons made in 1884 and exhibited in the Inventions Exhibition of 1885. Running at the extraordinary speed of 18,000 revolutions a minute, the turbine drove an armature of only $2 \frac{5}{8}$ in. diameter, the construction of which displayed as much ingenuity as the turbine itself. It was not mentioned by Sir Alfred, and it is little known, that among the important novelties in this machine was a system of forced lubrication.

No. 3264, VoL. 129] 